[AMERican Journal of Science, Vol. 314, November, 2014, P. 1284-1299, DOI 10.2475/09.2014.02]

\title{
SODIUM-POTASSIUM INTERDIFFUSION IN POTASSIUM-RICH ALKALI FELDSPAR I: FULL DIFFUSIVITY TENSOR AT $850^{\circ} \mathrm{C}$
}

\author{
ELENA PETRISHCHEVA* ${ }^{\dagger}$, RAINER ABART*, ANNE-KATHRIN SCHÄFFER*, \\ GERLINDE HABLER*, and DIETER RHEDE**
}

\begin{abstract}
Anisotropic diffusion is described by a tensor of diffusivities, $D_{\alpha \beta}$, which may be composition dependent leading to nonlinear anisotropic diffusion. In this work the practical problem of reconstructing such a tensor for $\mathrm{Na}-\mathrm{K}$ interdiffusion in potassium-rich alkali feldspar in the composition range $0.85 \leq X_{\mathrm{Or}} \leq 1.00$ is addressed. Gem quality sanidine with an initial composition of $X_{\mathrm{Or}}=0.85$ was exchanged with $\mathrm{KCl}$ salt melt at $850{ }^{\circ} \mathrm{C}$ and $\approx 1$ bar. The diffusivity tensor $D_{\alpha \beta}\left(X_{\mathrm{Or}}\right)$ and its composition dependence was reconstructed from composition profiles produced by cation exchange in six different crystallographic directions using a generalization of the Boltzmann approach. Na-K interdiffusion in potassium-rich alkali feldspar is considerably anisotropic and composition dependent. The principal axes of the diffusivity tensor representing the directions of highest and lowest diffusivity lie in the a-c plane with highest diffusivity parallel to the [101] direction, lowest diffusivity perpendicular to the $(10 \overline{1})$ plane, and the direction with intermediate diffusivity parallel to the crystallographic b-axis. All diffusivities $D_{\alpha \beta}\left(X_{O r}\right)$ increase as $X_{O r}$ tends to unity. Our main result is given in the form of numerical values for all components of the $D_{\alpha \beta}\left(X_{O r}\right)$ tensor for $0.85 \leq X_{\mathrm{Or}} \leq 1$.
\end{abstract}

Key words: Sodium-potassium, interdiffusion, potassium-rich alkali feldspar, anisotropy, diffusivity tensor, composition dependence

\section{INTRODUCTION}

Alkali feldspar is among the most abundant rock forming minerals in the Earth's crust. It forms a solid-solution series between the sodium $\left(\mathrm{NaAlSi}_{3} \mathrm{O}_{8}\right)$ and potassium $\left(\mathrm{KAlSi}_{3} \mathrm{O}_{8}\right)$ end-members. The interdiffusion of $\mathrm{Na}^{+}$and $\mathrm{K}^{+}$on the alkali sublattice plays a pivotal role in the re-equilibration of $\mathrm{Na}-\mathrm{K}$ partitioning between alkali feldspar and other Na- and K-bearing minerals in rocks undergoing pressure-temperature change (Voll and others, 1994). Knowledge of Na-K interdiffusion in alkali feldspar is crucial for the application of geo-thermobarometers such as the two feldspar thermometer (Fuhrman and Lindsley, 1988; Benisek and others, 2010) and for quantifying the kinetics of exsolution during cooling (Yund, 1984; Petrishcheva and Abart, 2009; Abart and others, 2009b; Abart and others, 2009a; Petrishcheva and Abart, 2012).

For alkali feldspar the Na-K interdiffusion coefficient can be calculated from the experimentally determined $\mathrm{Na}-$ and $\mathrm{K}$ - tracer diffusion coefficients according to Manning (1968)

$$
D_{N a K}=\frac{D_{N a}^{*}(c) D_{K}^{*}(c)}{(1-c) D_{N a}^{*}(c)+c D_{K}^{*}(c)}\left[1+\frac{\partial \ln \gamma_{c}}{\partial \ln c}\right]
$$

where $D_{\mathrm{NaK}}$ is the Na-K interdiffusion coefficient, and $c$ is a short hand notation for the orthoclase mole fraction in alkali feldspar $c \equiv X_{\mathrm{Or}}=\frac{K}{K+N a}$ in atomic units; $D_{N a}^{*}(c)$ and $D_{K}^{*}(c)$ are the sodium and the potassium tracer diffusion coefficients, respectively, which are considered as composition dependent; $\gamma_{c}$ is the activity coefficient of the

\footnotetext{
* Department of Lithospheric Research, University of Vienna, Althanstrasse 14, A-1090, Vienna, Austria

** Helmholtzzentrum Potsdam, Deutsches GeoForschungsZentrum, Telegraphenberg, D-14473, Potsdam, Germany

† Corresponding author: elena.petrishcheva@univie.ac.at
} 
potassium end-member phase component, which can be calculated from thermodynamic mixing models such as given by Hovis and others (1991). A number of tracer diffusion experiments have been done in which radioactive tracers including ${ }^{22} \mathrm{Na}$ (Lin and Yund, 1972; Foland, 1974; Kasper, ms, 1975), ${ }^{40} \mathrm{~K}$ (Lin and Yund, 1972), and ${ }^{41} \mathrm{~K}$ as a stable isotope tracer (Foland, 1974; Giletti and Shanahan, 1997) were used to determine the sodium and the potassium tracer diffusion coefficients in alkali feldspar. It was generally found that sodium diffuses much faster than potassium. In terms of the absolute values the results from the different studies show, however, considerable scatter (see the review by Cherniak, 2010), and calculation of Na-K interdiffusion coefficients from the respective tracer diffusion coefficients is rather uncertain. Alternatively, the Na-K interdiffusion coefficient can be determined directly from interdiffusion experiments. Thereby a diffusion couple consisting of two alkali feldspars of different compositions is annealed at elevated temperature to induce sodiumpotassium exchange. The interdiffusion coefficient is then obtained from the resulting composition profile across the interface between the two feldspars. The interdiffusion coefficient is generally expected to be composition dependent (Mehrer, 2007), which must be accounted for when determining the Na-K interdiffusion coefficient from composition-distance data. Christoffersen and others (1983) determined the Na-K interdiffusion coefficient in the composition range $0.1 \leq X_{\mathrm{Or}} \leq 0.8$ at temperatures of $900{ }^{\circ} \mathrm{C}$ and $1000{ }^{\circ} \mathrm{C}$ and at pressures of 0.5 and $1.5 \mathrm{GPa}$. They found a pronounced diffusion anisotropy, where diffusion perpendicular to (010) is about 12 times slower than perpendicular to (001). Moreover, a composition dependence with a minimum in $D_{\mathrm{NaK}}$ at $X_{\mathrm{Or}} \approx 0.4$ was documented. Comparison of the experimentally determined interdiffusion coefficients with those obtained from equation (1) using the experimentally determined Na- and K tracer diffusion coefficients of Kasper (ms, 1975) for albite and of Foland (1974) for orthoclase with $X_{\mathrm{Or}}=0.94$ delivered a similar composition dependence of $D_{\mathrm{NaK}}$ as experimentally determined. In terms of the absolute values the experimentally determined interdiffusion coefficients were, however, by a factor of 10 $(\perp(001))$ and $100(\perp(010))$ lower than predicted from equation $(1)$. The study of Christoffersen and others (1983) contributed substantially to the understanding of Na-K interdiffusion in alkali feldspar. Several questions remained unanswered though. In particular, the composition range of $0.8 \leq X_{\mathrm{Or}} \leq 1.0$ was not covered. This composition range is, however, of considerable interest, because most potassium-rich alkali feldspars in natural rocks fall into this composition range. Christoffersen and others (1983) give the interdiffusion coefficients for selected crystallographic directions, they do, however, not provide the full diffusivity tensor, which is necessary for a comprehensive description of the direction dependence of Na-K interdiffusion. Furthermore, it has been shown that localized, diffusion induced composition change in single crystal alkali feldspar produces coherency stress, which may eventually induce fracturing Petrović (ms, 1972); Neusser and others (2012); Scheidl and others (2014). According to Scheidl and others (2014) fracturing may occur, when composition shifts exceed about 10 mole percent. The feldspars in the diffusion couples of Christoffersen and others (1983) were shifted by about 50 mole percent close to the contact plane of the two feldspars. It is therefore possible that in the experiments of Christoffersen and others (1983) the composition induced coherency strain was accommodated by creep or by the formation of microcracks. The associated increase in defect density may have an influence on Na-K interdiffusion, but such effects were neither documented nor evaluated by the latter authors.

In this study we present the results of dedicated $\mathrm{Na}-\mathrm{K}$ interdiffusion experiments. In contrast to the study by Christoffersen and others (1983) we used a single crystal alkali feldspar and $\mathrm{KCl}$ salt melt as a diffusion couple, and composition shifts in the feldspar did not exceed 15 mole percent. That way mechanical effects due to large 
TABLE 1

Directions of measurement profiles and number of experiments done for different run durations

\begin{tabular}{|c|c|c|c|c|c|c|}
\hline direction & direction cosines & 1 day & 4 days & 8 days & 16 days & 32 days \\
\hline 1 & 100 & 2 & 1 & & & \\
\hline 2 & 010 & & 1 & 3 & 2 & 1 \\
\hline 3 & 001 & 3 & 2 & 2 & & \\
\hline 4 & $\frac{\sqrt{2}}{2} \frac{\sqrt{2}}{2} 0$ & & & 1 & 2 & \\
\hline 5 & $\frac{\sqrt{2}}{2} 0 \frac{\sqrt{2}}{2}$ & & & 1 & & \\
\hline 6 & $0 \frac{\sqrt{2}}{2} \frac{\sqrt{2}}{2}$ & & & 1 & & \\
\hline
\end{tabular}

composition shift in a single crystal feldspar and their potential feedback on interdiffusion could be minimized. The theory necessary for data reduction in the specific setting of composition-dependent, anisotropic Na-K interdiffusion in monoclinic alkali feldspar is presented and the full diffusivity tensor and its composition dependence in the composition range $0.85 \leq X_{\mathrm{Or}} \leq 1.0$ is derived for $850{ }^{\circ} \mathrm{C}$ and $\approx 1$ bar. The temperature dependence of $D_{\mathrm{NaK}}$ in the temperature range from $800{ }^{\circ} \mathrm{C}$ to $1000{ }^{\circ} \mathrm{C}$ and for compositions in the range $0.65 \leq X_{\mathrm{Or}} \leq 1.0$ for diffusion in the directions perpendicular to (001) and (010) are presented in the companion paper by Schäffer and others $(2014 b)$.

\section{EXPERIMENT}

\section{Cation Exchange Experiments}

Interdiffusion of $\mathrm{Na}^{+}$and $\mathrm{K}^{+}$in alkali feldspar was studied by means of cationexchange experiments, where $\mathrm{Na}^{+}$and $\mathrm{K}^{+}$ions were exchanged between alkali feldspar and $\mathrm{KCl}$ salt melt at $850{ }^{\circ} \mathrm{C}$ and $\approx 1$ bar. Gem quality sanidine from Volkesfeld (E-Eifel, Germany) with an original composition of $X_{\mathrm{Or}}=0.85$ was used as starting material. The sanidine is practically free of calcium and has minor Ba contents of up to 1.3 weight percent, $\mathrm{Fe}$ up to 0.2 weight percent, and $\mathrm{Sr}$ up to 0.2 weight percent (Demtröder, ms, 2011; Neusser and others, 2012). The sanidine is chemically homogeneous down to the nanometer scale. It is devoid of cracks, twins, exsolutions, second phase precipitates or other structural or chemical heterogeneities (Weitz, 1972; Riley and Bailey, 2003; Demtröder, ms, 2011; Neusser and others, 2012). Aluminum and silicon are highly disordered on the tetrahedral site with $\mathrm{t} 1=61$ (Neusser and others, 2012) resulting in monoclinic symmetry, space group $\mathrm{C} 2 / \mathrm{m}$.

Selected sanidine grains were oriented on a single crystal diffractometer and then machined to plates of about $3 \times 3 \times 1 \mathrm{~mm}$. The plates were cut in six different crystallographic orientations, and the $3 \times 3 \mathrm{~mm}$ surfaces were polished using diamond paste down to $1 \mu \mathrm{m}$ grain size on a rotary polisher. The feldspar plates were then put into quartz glass tubes with an inner diameter of $8 \mathrm{~mm}$ together with reagent grade $\mathrm{KCl}$ salt. The amount of $\mathrm{KCl}$ was chosen so as to achieve a molar ratio of 40:1 for the alkali cations contained in the salt and in the feldspar, respectively. That way an essentially constant composition of the salt melt was ensured during the exchange experiments. The quartz glass tubes were then sealed under vacuum and put into a pre-heated box furnace at $850{ }^{\circ} \mathrm{C}$ for 1 to 64 days (see table 1 ). The temperature was monitored with a type $\mathrm{N}$ thermocouple placed directly at the sample and was accurate to within $\pm 2^{\circ}$. It was shown by Neusser and others (2012) that the Al-Si ordering state does not change 

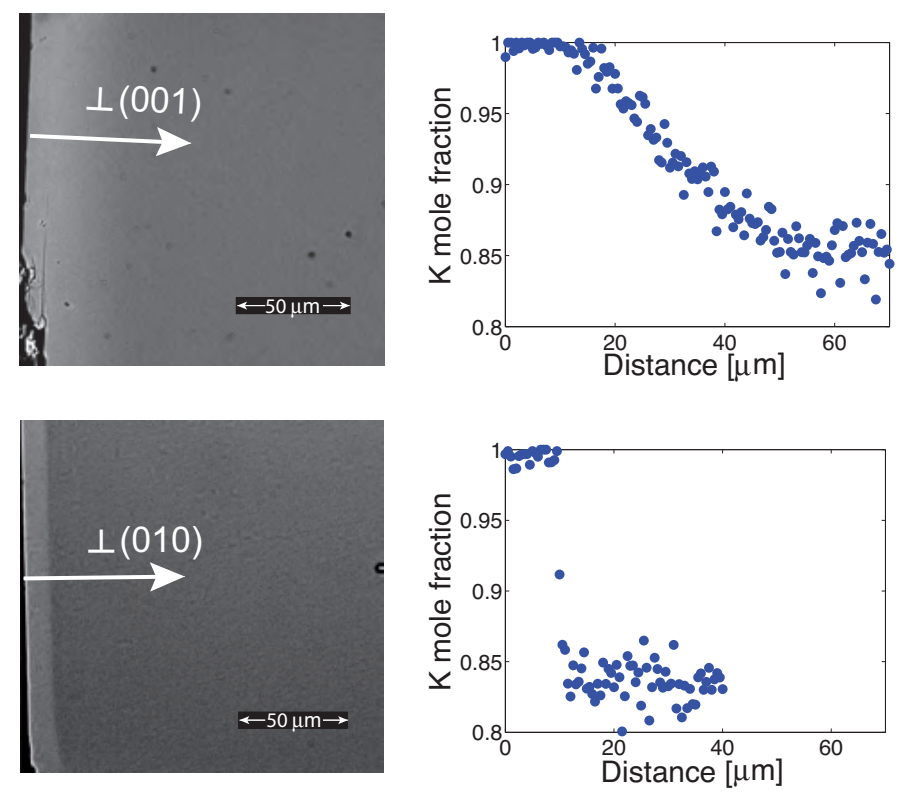

Fig. 1. Back-scatter electron images and composition-distance data for analysis profiles perpendicular to a polished (001) (top) and (010) (bottom) surface of sanidine after exchange with $\mathrm{KCl}$ melt at $850{ }^{\circ} \mathrm{C}$ for 8 days.

during cation exchange between sanidine and $\mathrm{KCl}$ salt melt at $850{ }^{\circ} \mathrm{C}$. After the experiment the samples were quenched within a few seconds by dropping into cold water. The tubes were opened and the feldspars were retrieved by dissolving the salt in deionized water. The feldspars retained their surface polish and no indications of surface reactions could be detected. The feldspar plates were then mounted in epoxy with the polished faces perpendicular to the surface of the mount. The samples were then cut, ground and polished mechanically using diamond paste down to $0.25 \mu \mathrm{m}$ grain size. Finally a chemomechanical polish using a silica suspension with basic $\mathrm{pH}$ was applied to remove the defect rich surface layer for later electron backscatter diffraction (EBSD) analysis.

\section{Scanning Electron Microscopy}

Scanning electron microscopy was done on a Quanta 3D FEG instrument at the Center of Earth Sciences, University of Vienna. Backscattered electron images were taken to document chemical alteration of the feldspar that is readily discerned from the change in gray shade on BSE images (fig. 1). In general, cation exchange between the sanidine and the $\mathrm{KCl}$ melt lead to a composition shift from the original composition of $X_{\mathrm{Or}}=0.85$ to pure potassium end-member composition with $X_{\mathrm{Or}}=1.00$. On the BSE images light gray zones of constant width are observed along the rims of the grains showing that chemical alteration starts from the grain surfaces and progresses into the grain interior with time. The exchanged outer domains of the grains and the internal domains with the original composition preserved are separated by more or less sharp transition zones (fig. 1). Chemical alteration within the single crystal sanidine requires that $\mathrm{Na}^{+}$and $\mathrm{K}^{+}$interdiffuse on the alkali sublattice of the feldspar, and the transition zone may be referred to as a diffusion front. Interestingly, the diffusion fronts are relatively sharp in [010]-direction and comparatively broad in the 
direction perpendicular to the (001) plane indicating diffusion anisotropy. In some cases, the lattice strain associated with cation exchange induced fracturing and produced irregularities in sample geometry and in the chemical pattern. Only those portions of the crystal were chosen for further mineral chemical analysis, where the diffusion fronts were undisturbed.

The crystal lattice orientation of the feldspar samples embedded in epoxy resin was determined by Electron backscatter diffraction analysis (EBSD). The Quanta 3D FEG instrument equipped with an EDAX Digiview IV EBSD camera was operated at 15 $\mathrm{keV}$ accelerating voltage and 2 to $3 \mathrm{nA}$ probe current in analytical mode. During analysis the sample surface was tilted to establish a beam incidence angle of 20 at a working distance of 10 to 14 millimeters. EBSD data collection and processing was performed using the EDAX OIM Data Collection and Analysis Software (versions 5.31 or 6.2). The background correction filter was generated on a natural polycrystalline alkali feldspar aggregate sample. To optimize the EBSD pattern contrast and intensity histogram normalization filter, a dynamic background subtraction filter and for some grains also a mean smoothing filter were applied. Due to partly weak EBSD patterns, the camera and Hough settings were adjusted for each grain separately. EBSD camera settings were at $2 \times 2$ binning using an exposure time of 300 to $400 \mathrm{msec}$. Hough settings of a binned pattern size of $160 \times 160$ pixels, a Theta step size of $0.5^{\circ}$ to $1^{\circ}$ and a $9 \times 9$ or $11 \times 11$ convolution mask were applied to a Rho-fraction of 72 percent to 85 percent for indexing at minimum 3 to 6 and at maximum 9 to 19 bands at a minimum distance of 8 to 17 pixels in Hough space. The orientation data were derived from $100 \times 100$ micrometer sized areas taken in a central portion of each crystal. Using step sizes of 10 to 25 micrometers in hexagonal grid mode yielded 23 to 126 data points per grain. In case of ambiguous results due to similar first and second solutions, single EBSD patterns were collected using 5 to 8 frames and evaluated manually for the different solutions in order to determine the actual orientation.

\section{Mineral Chemical Analysis}

Mineral chemical analyses were done on a field emission gun electron microprobe JEOL Hyperprobe JXA-8500F at Helmholtzzentrum Potsdam. Point analyses were taken along profiles from the polished sample surface inwards across the diffusion front and into the internal domains of the crystal. The exact orientation of each analysis profile with respect to the crystal lattice was reconstructed based on the EBSD data that were collected for each grain. Composition profiles were measured in six crystallographic directions that are given by the direction cosines with respect to the (a, b, $\left.{ }^{*}\right)$ frame: profile $1(1,0,0)$; profile $2(0,1,0)$; profile $3(0,0,1)$; profile $4(\sqrt{2} / 2$, $\sqrt{2} / 2,0)$; profile $5(\sqrt{2} / 2,0, \sqrt{2} / 2)$; profile $6(0, \sqrt{2} / 2, \sqrt{2} / 2)$ (see table 1$)$. Point analyses were taken at 0.5 to $1 \mu \mathrm{m}$ steps. The acceleration voltage and the beam current were set to $8 \mathrm{kV}$ and $10 \mathrm{nA}$, respectively. Counting times were 10 seconds on the peak and 5 seconds on the background on both sides of the peak. Short counting times were necessary to minimize loss of sodium by evaporation under the electron beam. Due to the small footprint of the beam and excitation volume, the analyses were very sensitive to imperfections of the sample surface such as scratches or beam induced carbon deposition. Despite the resulting scatter in the data, the analyzed trends clearly reflect the shapes of well defined diffusion profiles (fig. 2). The exchanged portions of the crystal attained pure potassium end-member composition. Corresponding to the variation in gray shade in the BSE image, the profile that evolved perpendicular to the (010) surface shows a relatively sharp transition zone between the outer exchanged domain and the grain interior with its original composition preserved. The sharp change in composition perpendicular to the (010) surface may give the impression of a dissolution-precipitation feature. The perfect preservation of the surface polish after cation exchange and the lack of evidence for surface reaction argue against this 


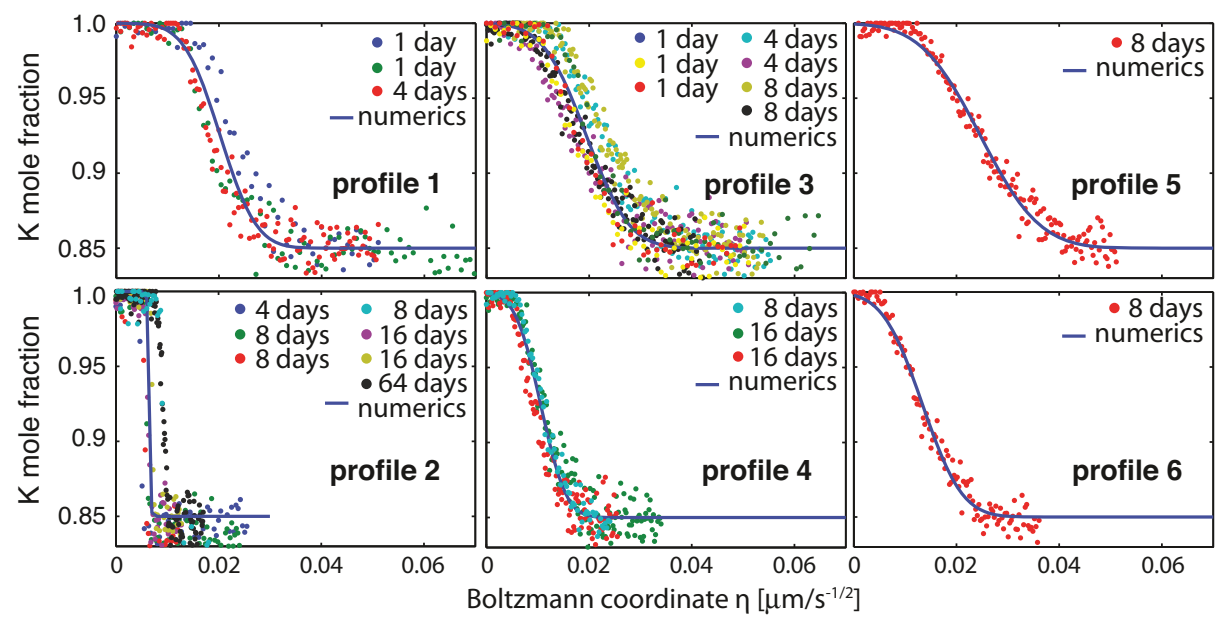

Fig. 2. Potassium mole fractions in alkali feldspar along different crystallographic directions versus rescaled Boltzmann coordinate $\eta$; direction cosines with respect to the $\left(\mathbf{a}, \mathbf{b}, \mathbf{c}^{*}\right)$ frame: profile $1(1,0,0)$; profile $2(0,1,0)$; profile $3(0,0,1)$; profile $4(\sqrt{2} / 2, \sqrt{2} / 2,0)$; profile $5(\sqrt{2} / 2,0, \sqrt{2} / 2)$; profile $6(0$, $\sqrt{2} / 2, \sqrt{2} / 2)$; different run-durations are indicated by different colors; after rescaling to $\eta$, all points from a certain direction approximately belong to the same curve; solid lines: mole fractions obtained from numerical solutions of equation (4) using the diffusivities determined from the experimental observations; the corresponding values of $D_{\alpha \beta}(c)$ are reported in figure 3 .

interpretation. Furthermore, in cation exchange experiments using similar experimental setup and starting material the Ba distribution remained unaffected across $\mathrm{Na}-\mathrm{K}$ exchange fronts (Neusser and others, 2012). In contrast, the $\mathrm{Ba}$ and also the $\mathrm{Fe}$ distribution in Eifel sanidine was completely changed across reaction fronts that developed during fluid induced albitization of Eifel sanidine by reaction with $\mathrm{NaCl}$ brine, which is clearly associated with dissolution-precipitation (Norberg and others, 2012) suggesting that dissolution-precipitation occurs when feldspar is reacted with a fluid but does not occur when sanidine is reacted with a "dry" alkali halide melt. Moreover, in an EBSD study on the lattice strain across a sharp concentration front along the [010] direction produced in Eifel sanidine during cation exchange in a similar experimental setup Schäffer and others (2014a) found localized elastic lattice strain but no indication for recrystallization across the sharp composition front, which further corroborates the interpretation of the observed composition contrasts as diffusion fronts. The transition between the exchanged external and the pristine internal portions of a grain is more gradual in the direction perpendicular to the $(001)$ plane than in [010] direction. In the profiles in both crystallographic directions a plateau is visible in the outer portion, where the composition is at $X_{\mathrm{Or}}=1.00$, and an inflection point is located in the transition zone. Given that the composition of the salt remained essentially unchanged during the cation exchange experiments, and $\mathrm{Na}-\mathrm{K}$ interdiffusion occurred under a constant concentration boundary condition at the crystal surface, such a front geometry can only be explained by a composition dependence of the interdiffusion coefficient. In the following we present the theory used for data reduction in the specific case of composition dependent anisotropic interdiffusion of $\mathrm{Na}^{+}$and $\mathrm{K}^{+}$in an alkali feldspar with monoclinic symmetry.

\section{THEORY}

A diffusive particle flux, further denoted by $\mathbf{j}(\mathbf{r}, t)$, results solely from the nonuniform distribution of the diffusant (Crank 1975). In what follows, the distribu- 
tion of the diffusant is denoted by $c(\mathbf{r}, t)$, the latter may refer to the, for example, volume concentration or mole-fraction, depending on the problem at hand. For our case $c(\mathbf{r}, t)$ is identical to $X_{\mathrm{Or}}(\mathbf{r}, t)$. The non-uniformity is characterized by the gradient vector $\nabla c(\mathbf{r}, t)=\left(\partial c / \partial x_{1}, \partial c / \partial x_{2}, \partial c / \partial x_{3}\right)$ pointing to where the concentration of the diffusant increases the most. The concept behind Fick's diffusion law is that in an isotropic medium the flux vector and the gradient vector are antiparallel and proportional to each other, $\mathbf{j}=-D \nabla c$. In the isotropic case the quantity $D$ is just a scalar quantity and is referred to as the diffusivity.

If the diffusivity depends on diffusant concentration, $D=D(c)$, this is referred to as nonlinear diffusion. Combining Fick's law with the general conservation law for the particle number

$$
\frac{\partial c}{\partial t}=-\nabla \mathbf{j}
$$

we obtain the diffusion equation

$$
\frac{\partial c}{\partial t}=\sum_{\alpha} \frac{\partial}{\partial x_{a}}\left[D(c) \frac{\partial c}{\partial x_{\alpha}}\right], \quad \alpha=1,2,3,
$$

where $\alpha$ denotes axes of an orthogonal coordinate frame chosen within the material.

If one knows $D(c)$, equation (3) can be discretized and solved numerically to obtain $c(\mathbf{r}, t)$ from $c(\mathbf{r}, 0)$ and boundary conditions. If $D(c)$ is unknown, one can address the inverse problem: obtain $D(c)$ from the experimentally measured $c(\mathbf{r}, t)$. An example thereof is the Boltzmann-Matano analysis (Matano, 1933).

In this section we use the above reported experimental data and reconstruct diffusivities for the anisotropic case. It is important to note that our geometry differs from that of the Boltzmann-Matano analysis. Therefore we first revisit the reconstruction scheme.

\section{Reduction}

In a crystal, where anisotropy is encountered, Fick's law remains valid, but becomes more complicated. The relation between the two vectors $\mathbf{j}$ and $\nabla c$ is described by a tensor (Crank, 1975)

$$
j_{\alpha}=-\sum_{\beta} D_{\alpha \beta} \frac{\partial c}{\partial x_{\beta}}, \quad \alpha, \beta=1,2,3,
$$

where the elements of the second-rank diffusivity tensor may depend on the diffusant concentration, $D_{\alpha \beta}=D_{\alpha \beta}(c)$. Then the diffusion equation equation (3) is replaced by

$$
\frac{\partial c}{\partial t}=\sum_{\alpha, \beta} \frac{\partial}{\partial x_{\alpha}}\left[D_{\alpha \beta}(c) \frac{\partial c}{\partial x_{\beta}}\right] .
$$

At a first glance, all directions are mixed in equation (4). Still, it is possible to reduce equation (4) to an effectively one-dimensional equation. To explain this we consider diffusive flow into a semi-infinite block, $x_{1} \geq 0$, consisting of an anisotropic solid with the boundary at $x_{1}=0$. Let us assume that $c=c\left(x_{1}, t\right)$. Even such a simple plane-parallel distribution leads to a three-component flux 


$$
\mathbf{j}=-\left(\begin{array}{ccc}
D_{11} & D_{12} & D_{13} \\
D_{21} & D_{22} & D_{23} \\
D_{31} & D_{32} & D_{33}
\end{array}\right)\left(\begin{array}{c}
\frac{\partial c}{\partial x_{1}} \\
0 \\
0
\end{array}\right)=-\left(\begin{array}{c}
D_{11} \frac{\partial c}{\partial x_{1}} \\
D_{21} \frac{\partial c}{\partial x_{1}} \\
D_{31} \frac{\partial c}{\partial x_{1}}
\end{array}\right) .
$$

Still only $\mathbf{j}_{x 1}=-D_{11}(c) \partial c / \partial x_{1}$ contributes to $\nabla \mathbf{j}$ in equation (2). Therefore the plane-parallel distribution evolves in full analogy with the simplest diffusion equation in one spatial dimension

$$
\frac{\partial c}{\partial t}=\frac{\partial}{\partial x_{1}}\left[D_{11}(c) \frac{\partial c}{\partial x_{1}}\right]
$$

Hence, if we prepared a large enough feldspar plate with one of the axes parallel to $O x_{1}$ and measured how the cations from the salt penetrate into the crystal along this axis, we would get $c\left(x_{1}, t\right)$. We then could retrieve $D_{11}(c)$.

This approach can be extended to recover the full tensor $D_{\alpha \beta}(c)$. Consider diffusion along some arbitrary direction given by a unit vector $\mathbf{n}$ with the components (direction cosines) $n_{1}, n_{2}$, and $n_{3}$. The plane-parallel diffusant distribution is given by $c=c(\xi, t)$, where the "propagation coordinate" $\xi$ is defined as

$$
\xi=n_{1} x_{1}+n_{2} x_{2}+n_{3} x_{3},
$$

and the origin of the $\left(x_{1}, x_{2}, x_{3}\right)$ frame is taken at the salt-crystal interface, such that the boundary conditions are specified at $\xi=0$. Using the chain rule

$$
\frac{\partial}{\partial x_{\alpha}}=n_{\alpha} \frac{\partial}{\partial \xi}
$$

it is easy to see that equation (5) is generalized to the form

$$
c=c(\xi, t), \quad \frac{\partial c}{\partial t}=\frac{\partial}{\partial \xi}\left[D_{n}(c) \frac{\partial c}{\partial \xi}\right],
$$

where $D_{n}(c)$ denotes the scalar diffusivity along the direction $\mathbf{n}$

$$
D_{n}(c)=\sum_{\alpha, \beta} D_{\alpha \beta}(c) n_{\alpha} n_{\beta}
$$

Equation (6) is naturally coupled with the following initial and boundary conditions

$$
\xi \geq 0,\left.\quad c(\xi, t)\right|_{\xi=0}=\left.c_{1} \quad c(\xi, t)\right|_{t=0}=c_{2},
$$

where $c_{1}$ and $c_{2}$ are the potassium mole fraction of the alkali feldspar in equilibrium with the salt melt, and the initial potassium mole fraction in the crystal, respectively. Now, if we recover $D_{n}(c)$ for a large enough number of directions, we can consider equation (7) as a system of linear equations that delivers the unknowns $D_{\alpha \beta}(c)$.

According to Onsager's principle $D_{\alpha \beta}=D_{\beta \alpha}$ so that only six diffusivities are left. Moreover, $D_{\alpha \beta}$ is affected by symmetries of the crystal. For the monoclinic symmetry at hand, we chose the $(\mathbf{a}, \mathbf{b}, \mathbf{c} *)$ frame in which $\mathbf{b}$ is set parallel to the crystallographic dyad axis. The diffusivity tensor then takes the form

$$
D_{\alpha \beta}=\left(\begin{array}{ccc}
D_{11} & 0 & D_{13} \\
0 & D_{22} & 0 \\
D_{31} & 0 & D_{33}
\end{array}\right)
$$


so that only four unknown diffusivities are left. However, to improve the accuracy, we considered six directions and measured up to seven profiles $c(\xi, t)$ for each direction (see table 1 ). The components $D_{\alpha \beta}$ were then obtained from Eq. (7) using the least squares method.

\section{Boltzmann's Method}

In this section we explain how $D_{n}(c)$ is recovered from the experimentally measured concentration distance data, that is solutions $c(\xi, t)$ of equations (6) to (8). The latter system has an important feature first noticed by Boltzmann (1894). Namely, one can introduce a combined space-time variable

$$
\eta=\frac{\xi}{2 \sqrt{t}} \quad \text { defined for } t>0,
$$

and look for a special class of solutions of the diffusion equation: functions $c(\xi, t)$ that are reduced to a composite function $c(\eta)$ of a single variable $(9)$. It is easy to see that for such solutions the nonlinear diffusion equation (6) transforms to

$$
-2 \eta \frac{d c}{d \eta}=\frac{d}{d \eta}\left[D_{n}(c) \frac{d c}{d \eta}\right], \quad 0 \leq \eta<+\infty
$$

that is an ordinary differential equation. The key question is: are the initial and boundary conditions compatible with the ansatz $c=c(\eta)$ ? For instance, this is the case for the Boltzmann-Matano analysis. Our geometry is different but still compatible with the Boltzmann variable. From equation (8) we obtain that

$$
\left.c(\eta)\right|_{\eta=0}=c_{1} \text { and }\left.c(\eta)\right|_{\eta=+\infty}=c_{2} .
$$

Therefore, the initial value problem for the partial differential system (6) to (8) exactly reduces to a boundary value problem for an ordinary differential system (10) to (11).

We now turn to the inverse problem and express $D(c)$ in terms of $c(\eta)$. To this end we integrate equation (10) over $d \eta$ over the interval $[\eta, \infty]$. Note, that it is natural to assume that $c^{\prime}(\eta)=d c / d \eta \rightarrow 0$ for $\eta \rightarrow \infty$. Therefore

$$
2 \int_{\eta}^{\infty} \eta c^{\prime}(\eta) d \eta=\left.c^{\prime}(\eta) D_{n}(c)\right|_{c=c(\eta)}
$$

Finally we note that with the increase of $\eta, c(\eta)$ decreases monotonically from $c(0)=c_{1}$ to $c(\infty)=c_{2}$. Therefore one can introduce the inverse function $\eta=\eta(c)$ and transform equation (12) to the form

$$
D_{n}(c)=-2 \eta^{\prime}(c) \int_{c_{2}}^{c} \eta(c) d c .
$$

In what follows equation (13) is used for calculating $D(c)$.

\section{Results for Diffusivities}

The results from all interdiffusion experiments which were done along six different crystallographic directions are shown in figure 2. Let $\left(\xi_{s}, c_{s}\right), 1 \leq s \leq S$ denote experimental observations of the mole fraction profile $c=c(\xi, t)$ for some fixed direction $\mathbf{n}$ and time $t=t_{0}$. First, the space coordinate was rescaled to $\eta_{s}=\xi_{s} /\left(2 \sqrt{t_{0}}\right)$ and then the points $\left(\eta_{s}, c_{s}\right)$ obtained from all experiments done along this specific direction were plotted together (see fig. 2). Although the measurements had been 


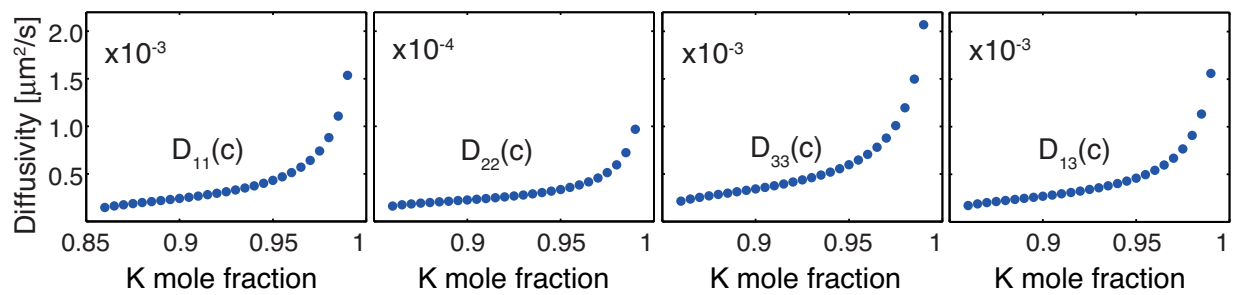

Fig. 3. Nonzero components of the diffusivity tensor $D_{\alpha \beta}(c)$ versus mole fraction of $\mathrm{K}$ cations in feldspar. The indices $1 \leq \alpha, \beta \leq 3$ refer to the $\left(\mathbf{a}, \mathbf{b}, \mathbf{c}^{*}\right)$ frame in which $\mathbf{b}$ is set parallel to the crystallographic dyad axis such that both $D_{12}$ and $D_{23}$ vanish.

done for several values of $t_{0}$, all rescaled points approximately belong to the same curve. The latter represents $c(\eta)$ for the fixed direction $\mathbf{n}$. In a second step, the data were smoothed assigning the same weight to each of the profiles measured along one direction. The smoothed data set $\left(\eta_{s}, c_{s}\right)$ was then used to derive the inverse function $\eta(c)$. The latter was inserted into equation (13) to obtain $D_{n}(c)$. We calculated $D_{n}(c)$ for six directions, each one with up to seven values of $t_{0}$. Finally equation (7) was solved with respect to $D_{\alpha \beta}(c)$ to recover the unknown components of the diffusivity tensor. The results for the components of $D_{\alpha \beta}(c)$ are shown in figure 3 , and the numerical values are given in table 2. Finally we tested our results by direct calculation of the numerical solutions of the nonlinear diffusion equation (4) with the previously extracted composition-dependent diffusivities. Both the boundary and the initial conditions reflect the experimental setup. The numerical results are shown by the solid lines in figure 2. A reasonable agreement with the actually observed compositiondistance data (points in fig. 2) was found. Based on our results the Na-K interdiffusion coefficient for $850^{\circ} \mathrm{C}$ can now be calculated for any direction in alkali feldspar using equation (7).

One immediately observes that Na-K interdiffusion is composition dependent, see figure 3 . At close to potassium end-member composition $X_{\mathrm{Or}} \geq 0.95$ a small decrease of $X_{\mathrm{Or}}$ leads to a quick decrease of all diffusivities. In the composition range $0.85 \leq X_{\mathrm{Or}} \leq$ 0.95 decrease of $D_{\mathrm{NaK}}$ still leads to decrease of diffusivities, the effect is however less pronounced. Furthermore, Na-K interdiffusion is markedly anisotropic. The diffusion anisotropy is best visualized by plotting characteristic surfaces for the tensor $D_{\alpha \beta}(c)$. Four exemplary values of the potassium mole fraction were used, see figure 4 . We recall (Nye, 1957) that the characteristic ellipsoid shows how diffusivity depends on direc-

TABLE 2

Nonzero components of the diffusivity tensor $D_{\alpha \beta}(c)$

\begin{tabular}{lccccc}
\hline K mole fraction & 0.86 & 0.875 & 0.89 & 0.905 & 0.92 \\
\hline \hline D11 $\left[\mu \mathrm{m}^{2} / \mathrm{s}\right]$ & $1.5 \times 10^{-4}$ & $1.9 \times 10^{-4}$ & $2.2 \times 10^{-4}$ & $2.5 \times 10^{-4}$ & $3.0 \times 10^{-4}$ \\
D22 $\left[\mu \mathrm{m}^{2} / \mathrm{s}\right]$ & $1.6 \times 10^{-5}$ & $1.9 \times 10^{-5}$ & $2.1 \times 10^{-5}$ & $2.3 \times 10^{-5}$ & $2.6 \times 10^{-5}$ \\
$\mathrm{D} 33\left[\mu \mathrm{m}^{2} / \mathrm{s}\right]$ & $2.1 \times 10^{-4}$ & $2.7 \times 10^{-4}$ & $3.1 \times 10^{-4}$ & $3.6 \times 10^{-4}$ & $4.2 \times 10^{-4}$ \\
D13 $\left[\mu \mathrm{m}^{2} / \mathrm{s}\right]$ & $1.7 \times 10^{-4}$ & $2.1 \times 10^{-4}$ & $2.5 \times 10^{-4}$ & $2.8 \times 10^{-4}$ & $3.2 \times 10^{-4}$ \\
\hline $\mathrm{K} \mathrm{mole} \mathrm{fraction}$ & 0.935 & 0.95 & 0.965 & 0.98 & 0.99 \\
\hline D11 $\left[\mu \mathrm{m}^{2} / \mathrm{s}\right]$ & $3.5 \times 10^{-4}$ & $4.3 \times 10^{-4}$ & $5.7 \times 10^{-4}$ & $8.8 \times 10^{-4}$ & $15.3 \times 10^{-4}$ \\
D22 $\left[\mu \mathrm{m}^{2} / \mathrm{s}\right]$ & $2.9 \times 10^{-5}$ & $3.4 \times 10^{-5}$ & $4.2 \times 10^{-5}$ & $5.9 \times 10^{-5}$ & $9.7 \times 10^{-5}$ \\
D33 $\left[\mu \mathrm{m}^{2} / \mathrm{s}\right]$ & $4.9 \times 10^{-4}$ & $6.0 \times 10^{-4}$ & $7.8 \times 10^{-4}$ & $12.0 \times 10^{-4}$ & $20.1 \times 10^{-4}$ \\
D13 $\left[\mu \mathrm{m}^{2} / \mathrm{s}\right]$ & $3.8 \times 10^{-4}$ & $4.6 \times 10^{-4}$ & $6.0 \times 10^{-4}$ & $9.1 \times 10^{-4}$ & $15.6 \times 10^{-4}$ \\
\hline
\end{tabular}




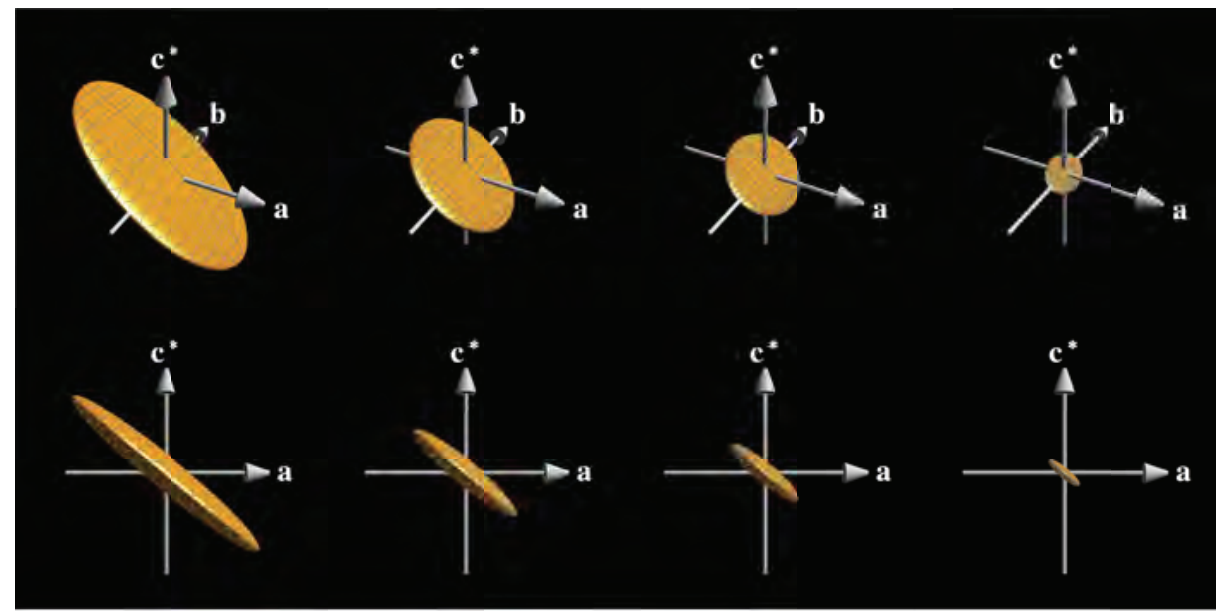

Fig. 4. Four examples of characteristic surfaces (from left to the right) for $X_{\text {Or }}=0.86,0.9,0.95,0.99$ representing the composition dependent diffusivity tensor; note that $D_{\mathrm{NaK}}$ in a certain direction is inversely proportional to the length of the ellipses radius vector in this direction.

tion: the larger the diffusivity in some direction, the more oblate-shaped is the ellipsoid in this direction. Na-K interdiffusion is represented by a three-axed ellipsoid with the intermediate principal axis parallel to the [010] direction and the shortest and longest principal axes in the (010) plane. The shortest principle axis of the representative ellipsoid encloses an angle of $50^{\circ}$ with the positive a-axis measured towards the positive $c^{*}$-axis. This direction exactly coincides with the crystallographic [101] direction indicating that the strongest diffusion anisotropy is in the (010) plane with fastest diffusion in the [101] direction and slowest diffusion in the direction within (010) that is perpendicular to [101]. The diffusion anisotropy is most pronounced for smaller values of the mole fraction $\left(X_{\mathrm{Or}} \approx 0.87\right)$ and is less pronounced close to potassium end-member compositions $\left(X_{\mathrm{Or}} \approx 1.00\right)$.

\section{DISCUSSION}

The most remarkable results of our study are the marked diffusion anisotropy and the composition dependence of Na-K interdiffusion at $X_{\mathrm{Or}} \geq 0.85$.

The observed diffusion anisotropy agrees well with what is expected from the crystal structure. Alkali feldspar contains wide channels parallel to the crystallographic [101] direction with the shortest diameter being about $4.5 \AA$, along which the alkali cations are aligned. By combining dielectric spectroscopy and atomistic computer simulation Jones and others (2004) identified these channels as the most likely pathways for the migration of $\mathrm{Na}^{+}$and $\mathrm{K}^{+}$cations by vacancy mediated conventional hopping giving raise to substitutional interdiffusion. These channels lie in the $(010)$ plane and enclose an angle of $50^{\circ}$ with the crystallographic a-axis measured from the positive a-axis towards the positive c-axis. This is exactly parallel to the principle axis of the experimentally determined diffusivity tensor that represents the fastest diffusion direction (fig. 4). No comparatively wide channels that would be suitable for alkali diffusion exist along other directions within the (010)-plane nor along the [010] direction. The anisotropy of Na-K interdiffusion becomes less accentuated at close to potassium end-member compositions. This is also the composition region with the strongest increase of $D_{\mathrm{NaK}}$.

A composition dependence of the Na-K interdiffusion coefficient was already suggested by earlier workers. Using similar cation exchange experiments as described 
in this study, Petrović (ms, 1972) found a substantial increase of $D_{\mathrm{NaK}}$ with increasing $X_{\mathrm{Or}}$ at compositions with $X_{\mathrm{Or}} \geq 0.86$, which is in line with our observations. With regard to the direction dependence, Petrović (ms, 1972), however, found Na-K interdiffusion to be quasi isotropic within the (010) plane and two orders of magnitude slower in [010] direction than within the (010) plane. From cation exchange experiments between alkali feldspar and $\mathrm{NaCl}-\mathrm{KCl}$ salt melt Neusser and others (2012) found that in potassium-rich alkali feldspar $D_{\mathrm{NaK}}$ increases with $X_{\mathrm{Or}}$, but $D_{\mathrm{NaK}}$ was not quantified. In the context of homogenization experiments on synthetic and natural cryptoperthite Brady and Yund (1983) remarked that considerable composition dependence of the interdiffusion coefficient may be expected at physical conditions where a solid-solution deviates significantly from a thermodynamic ideal solution. This notion is also inherent in exsolution by spinodal decomposition and can be predicted from theoretical considerations (Petrishcheva and Abart, 2009, 2012). It is also manifest in equation (1), in which the expression in parentheses corresponds to the "thermodynamic term" accounting for the non-ideality of the solution phase. Christoffersen and others (1983) compared their direct determinations of $D_{\mathrm{NaK}}$ from classical diffusion couple experiments at $1000^{\circ} \mathrm{C}$ to predictions based on equation (1) and indeed found a minimum in $D_{\mathrm{NaK}}$ at $X_{\mathrm{Or}} \approx 0.4$ as is predicted from equation (1), if the non-ideality of the alkali feldspar solid-solution is taken into account. These latter authors inferred a diffusion anisotropy with Na-K interdiffusion being 12 times faster perpendicular to (001) than perpendicular to (010). They also found a diffusion anisotropy within the (010) plane with Na-K interdiffusion in [100] direction being six times slower than perpendicular to $(001)$. Both these observations are in reasonable agreement with our findings.

A comparison of our experimentally determined Na-K interdiffusion coefficient and predictions based on equation (1) using experimentally determined tracer diffusion coefficients is shown in figure 5 . The non-ideality of the alkali feldspar solid-solution is most pronounced at intermediate compositions, it cannot, however, explain the strong composition dependence of the Na-K interdiffusion coefficient at close to potassium end-member composition. The steep raise of $D_{\mathrm{NaK}}$ with increasing $X_{\text {Or }}$ at potassium-rich compositions is mainly due to the fact that the potassium tracer diffusion coefficient is substantially lower than the sodium tracer diffusion coefficient. It must be noted that the tracer diffusion coefficients for sodium and potassium in albite by Kasper (ms, 1975) and in orthoclase by Foland (1974) were determined from bulk exchange experiments, and only the potassium tracer diffusion coefficient in albite from Giletti and Shanahan (1997) was measured parallel to the [010] direction. Irrespective of the choice of the tracer diffusion coefficients, our direct determinations of $D_{\mathrm{NaK}}$ are lower than the predictions from equation (1) by a factor of 3 to 5 for $D_{\mathrm{NaK}}^{\perp(001)}$ and by more than an order of magnitude for $D_{\mathrm{NaK}}^{\perp(010)}$. A reasonable agreement between theoretical prediction and experimental determination is obtained, if diffusion in the fastest direction, that is, $D_{\mathrm{NaK}}^{\max }$ is used for comparison. For further comparison of the interdiffusion model expressed by equation (1) and our direct determinations, the sodium and potassium tracer diffusion coefficients were back-calculated from $D_{\mathrm{NaK}}(c)$ assuming that they are independent of $X_{\text {Or }}$ (fig. 5). There is reasonable agreement between our back-calculated and the experimentally determined tracer diffusion coefficients for the direction of fastest diffusion, but our back-calculated values are substantially slower for the [010] direction (fig. 6). This is probably due to the fact that the tracer diffusion coefficients obtained from bulk exchange experiments largely represent the fastest diffusion direction. There is reasonably good agreement between our back-calculated tracer diffusion coefficient for potassium in the [010] direction and the corresponding value given by Giletti and Shanahan (1997) (fig. 6). Potassium tracer diffusion perpendicular to (010) has been estimated to be 0.1 to 0.6 times as slow 


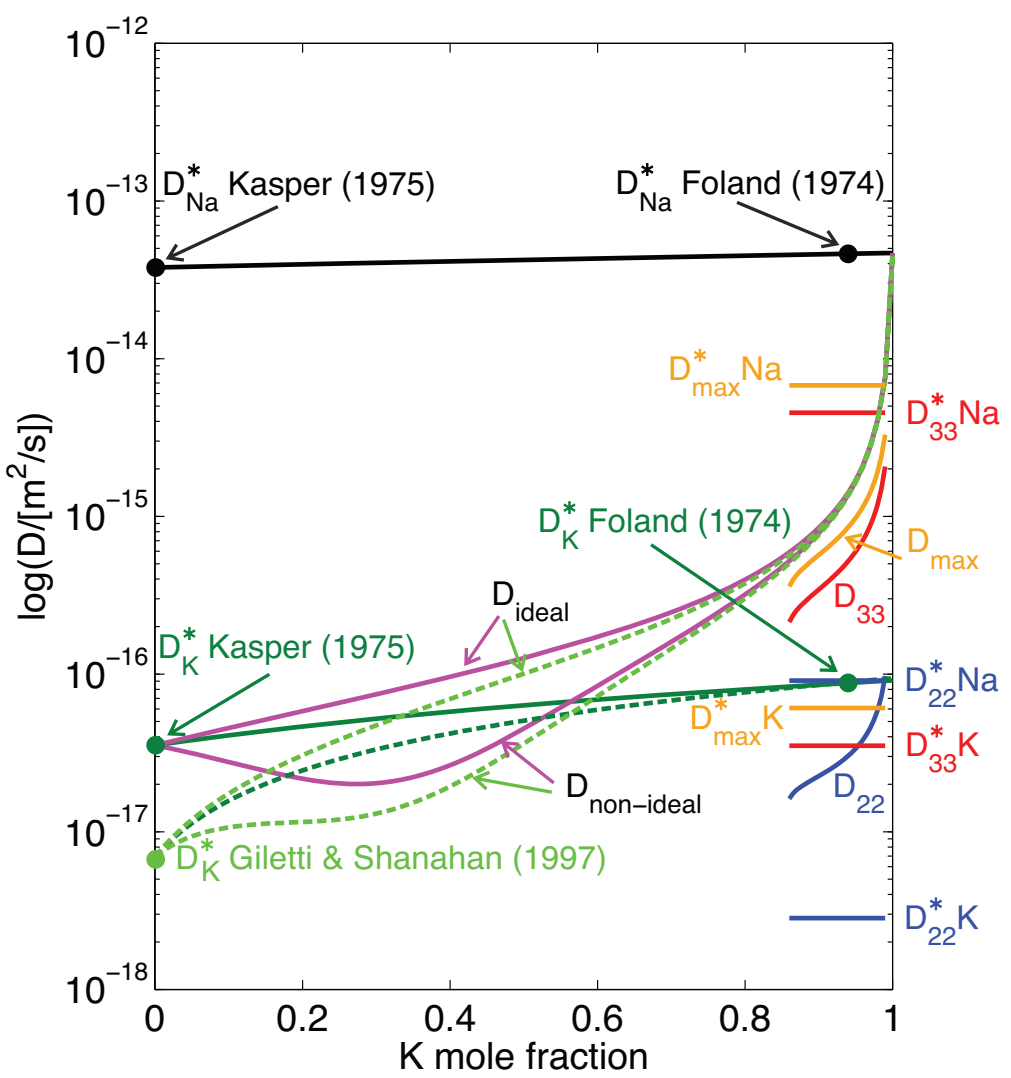

Fig. 5. Comparison of direct determinations of the Na-K interdiffusion coefficient and predictions based on equation (1) and using experimentally determined Na- and K-tracer diffusion coefficients of Kasper (ms, 1975) and Giletti and Shanahan (1997) for albite and of Foland (1974) for orthoclase, and the thermodynamic mixing model for the disordered alkali feldspar solid-solution of Hovis and others (1991); orange, red and blue curves represent our experimental determinations of the Na-K interdiffusion coefficient in the fastest diffusion direction, perpendicular to $(001)$ and perpendicular to $(010)$, respectively; the horizontal lines in similar colors represent the back-calculated tracer diffusion coefficients.

as perpendicular to (001) by Bailey (1971) and Giletti and others (1974), which is in line with our back-calculated potassium tracer diffusion coefficients.

Finally, it must be noted that the concentration-distance data we used for determining the diffusivity tensor reflect the effective diffusivities in the sense that all effects potentially influencing the interdiffusion of $\mathrm{Na}$ and $\mathrm{K}$, in particular its composition dependence and anisotropy, are integrated into the obtained diffusivities. The influence of deviations from thermodynamic ideal mixing behavior of the alkali feldspar solid-solution can safely be excluded, as the non-ideality of disordered alkali feldspar at $850{ }^{\circ} \mathrm{C}$, at $\approx 1$ bar and in the composition range of $X_{\mathrm{Or}} \geq 0.85$ is minute (fig. 5). Another potential influence is the coherency strain associated with compositional heterogeneities in a single crystal of alkali feldspar (Petrović, ms, 1972; Neusser and others, 2012; and Scheidl and others, 2014). On the one hand, the elastic stress associated with coherency influences cation interdiffusion via the stress dependence of the chemical potentials of the diffusing components (Larche and Cahn, 1982; Svoboda and others, 2002). On the other hand, the crystal lattice may be appreciably distorted across sharp composition fronts such as those observed along the [010] direction 


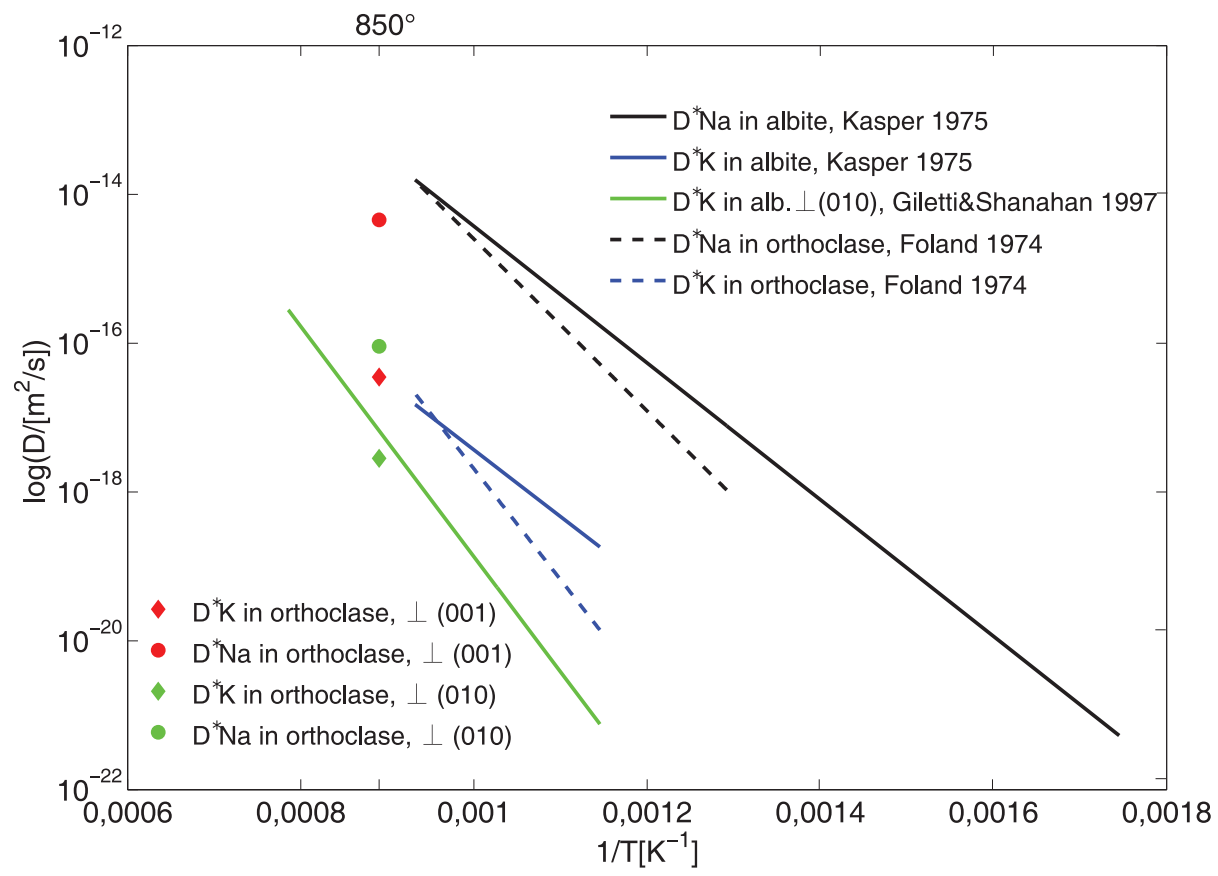

Fig. 6. Arrhenius diagram showing experimentally determined $\mathrm{Na}$ and $\mathrm{K}$ tracer diffusion coefficients from the literature (solid and dashed lines) and the $\mathrm{Na}$ and $\mathrm{K}$ tracer diffusion coefficients calculated from the composition dependence of $D_{\mathrm{NaK}}$ at $850{ }^{\circ} \mathrm{C}$ using equation (1) for two crystallographic directions (circular and diamond symbols).

(Schäffer and others, 2014a), and this lattice distortion may influence the ease of atomic jumps and thus Na-K interdiffusion in these domains. Boltzmann's approach, which we used for extracting diffusivities from composition-distance data, does not make any assumptions regarding these potential effects. It is independent of the microscopic mechanisms underlying the diffusion process, or of charge balance and crystal chemical constraints such as implicit in equation (1) (Manning, 1968). The reasonable agreement between the model predictions and our experimental determinations suggests that, within the limited scrutiny of the available experimental data, equation (1) is a useful model for Na-K interdiffusion in alkali feldspar. The above mentioned effects influencing $\mathrm{Na}-\mathrm{K}$ interdiffusion are probably of second order within the accuracy of the available calibrations of $\mathrm{Na}$ and $\mathrm{K}$ tracer diffusion. Nevertheless, theoretical calculations of $\mathrm{Na}-\mathrm{K}$ interdiffusion coefficients based on equation (1) should be applied with care. In particular, it remains to be seen, whether new, direction specific determinations of the respective tracer diffusion coefficients do show the according anisotropy needed to produce the observed direction dependence of the Na-K interdiffusion if inserted into equation (1).

\section{CONCLUSIONS}

The interdiffusion of $\mathrm{Na}^{+}$and $\mathrm{K}^{+}$in disordered, monoclinic alkali feldspar was investigated by cation exchange experiments using gem quality alkali feldspar single crystals with well defined geometry and a $\mathrm{KCl}$ salt melt as a diffusion couple at $850{ }^{\circ} \mathrm{C}$ and $\approx 1$ bar. Na-K interdiffusion in alkali feldspar is markedly anisotropic and composition dependent. The full diffusivity tensor was extracted in the composition range $0.85 \leq X_{\mathrm{Or}} \leq 1.00$. The strongest diffusion anisotropy is within the (010) plane 
with the principle axis of the diffusivity tensor representing fastest diffusion parallel to the [101] direction and slowest diffusion in the direction perpendicular to the $(10 \overline{1})$ plane. The principle axis corresponding to intermediate diffusivity is parallel to the crystallographic $\mathbf{b}$ axis. The direction of fastest diffusion is parallel to large channels in the framework structure of the feldspar that run in [101] direction. Based on our results the Na-K interdiffusion coefficient at $850{ }^{\circ} \mathrm{C}$ can now be calculated for any direction in alkali feldspar. The interdiffusion coefficients show a weak increase with increasing $X_{\mathrm{Or}}$ in the composition range $0.85 \leq X_{\mathrm{Or}} \leq 0.95$. At $X_{\mathrm{Or}} \geq 0.95$ the composition dependence is much more pronounced, and $D_{\mathrm{NaK}}$ strongly increases with increasing $X_{\text {Or }}$. This renders alkali feldspar with close to potassium end-member composition $\left(X_{\mathrm{Or}}>0.95\right)$ particularly sensitive to re-equilibration during slow cooling.

\section{ACKNOWLEDGMENTS}

We thank Susan Schorr and Juergen Schreuer for providing gem quality sanidines from the Eifel, Gerald Giester for orienting the feldspar crystals on a three circle goniometer, and Andreas Wagner for preparing the single crystal plates with polished surfaces. We are indebted to D. J. Cherniak and an R. Dohmen for their constructive comments on an earlier version of the manuscript. This work was funded by the Austrian Science Foundation, FWF grant I 474-N19 in the framework of the DFG-FWF research group FOR 741 and by the University of Vienna in the framework of the IK052 doctoral school.

\section{REFERENCES}

Abart, R., Petrishcheva, E., Kaessner, S., and Milke, R., 2009a, Perthite microstructure in magmatic alkali feldspar with oscillatory zoning; Weinsberg Granite, Upper Austria: Mineralogy and Petrology, v. 97, n. 3-4, p. 251-263, http://dx.doi.org/10.1007/s00710-009-0090-1

Abart, R., Petrishcheva, E., Wirth, R., and Rhede, D., 2009b, Exsolution by spinodal decomposition II: Perthite formation during slow cooling of anatexites from Ngoronghoro, Tanzania: American Journal of Science, v. 309, n. 6, p. 450-475, http://dx.doi.org/10.2475/06.2009.02

Bailey, A., 1971, Comparison of low-temperature with high-temperature diffusion of sodium in albite: Geochimica et Cosmochimica Acta, v. 35, n. 10, p. 1073-1081, http://dx.doi.org/10.1016/00167037(71)90023-8

Behrens, H., Johannes, W., and Schmalzried, H., 1991, On the mechanics of cation diffusion processes in ternary feldspars: Physics and Chemistry of Minerals, v. 17, n. 1, p. 62-78, http://dx.doi.org/10.1007/ BF00209227

Benisek, A., Dachs, E., and Kroll, H., 2010, A ternary feldspar-mixing model based on calorimetric data: development and application: Contributions to Mineralogy and Petrology, v. 160, n. 3, p. 327-337, http://dx.doi.org/10.1007/s00410-009-0480-8

Boltzmann, L., 1894, Zur Integration der Diffusionsgleichung bei variablen Diffusionskoeffizienten: Annalen der Physik, v. 289, p. 959-964, http://dx.doi.org/10.1002/andp.18942891315

Brady, J. B., and Yund, R. A., 1983, Interdiffusion of $\mathrm{K}$ and $\mathrm{Na}$ in alkali feldspars: homogenization experiments: American Mineralogist, v. 68, n. 1-2, p. 106-111.

Cherniak, D., 2010, Cation diffusion in feldspars: Reviews in Mineralogy and Geochemistry, v. 72, n. 1, p. 691-733, http://dx.doi.org/10.2138/rmg.2010.72.15

Christoffersen, R., Yund, R., and Tullis, J., 1983, Inter-diffusion of K and $\mathrm{Na}$ in alkali feldspars: diffusion couple experiments: American Mineralogist, v. 68, n. 11-12, p. 1126-1133.

Crank, J., 1975, The Mathematics of Diffusion: Oxford, Oxford University Press, 424 p.

Demtröder, K., ms, 2011, Untersuchung zur Al/Si-Ordnung an Sanidin Megakristallen aus der Eifel: Bochum, Germany, Ruhr-Universität Bochum, Master's thesis.

Foland, K., 1974, Alkali diffusion in orthoclase, in Hofmann, A. W., Giletti, B J., Yoder, H. S., and Yund, R. A., editors, Geochemical Transport and Kinetics: Washington, D.C., Carnegie Institution of Washington Publication 634, p. 77-98.

Fuhrman, M., and Lindsley, D., 1988, Ternary-feldspar modeling and thermometry: American Mineralogist, v. 73 , n. $3-4$, p. $201-215$.

Giletti, B. J., and Shanahan, T. M., 1997, Alkali diffusion in plagioclase feldspar: Chemical Geology, v. 139, n. 1-4, p. 3-20, http://dx.doi.org/10.1016/S0009-2541(97)00026-0

Giletti, B., Semet, M., and Kasper, R., 1974, Self-diffusion of potassium in low albite using an ion microprobe: Geological Society of America Abstracts with Programs, 6, p. 754.

Hovis, G. L., Delbove, F., and Bose, M. R., 1991, Gibbs energies and entropies of K-Na mixing for alkali feldspar from phase equilibrium data: implications for feldspar solvi and short range order: American Mineralogist, v. 76, p. 913-927.

Jones, A., Islam, M. S., Mortimer, M., and Palmer, D., 2004, Alkali ion migration in albite and K feldspar: 
Physics and Chemistry of Minerals, v. 31, n. 5, p. 313-320, http://dx.doi.org/10.1007/s00269-0040389-8

Kasper, R., ms, 1975, Cation and Oxygen diffusion in albite: Providence, Rhode Island, Brown University, Ph. D. Dissertation, $298 \mathrm{p}$.

Larche, F., and Cahn, J., 1982, The effect of self-stress on diffusion in solids: Acta Metallurgica, v. 30, n. 10, p. 1835-1845, http://dx.doi.org/10.1016/0001-6160(82)90023-2

Lin, T., and Yund, R., 1972, Potassium and sodium self-diffusion in alkali feldspar: Contributions to Mineralogy and Petrology, v. 34, n. 3, p. 177-184, http://dx.doi.org/10.1007/BF00373289

Manning, J. R., 1968, Diffusion kinetics for atoms in crystals: Princeton, New Jersey, D. Van Norstrand Company, Inc., $257 \mathrm{p}$.

Matano, C., 1933, On the relation between the diffusion-coefficients and concentrations of solid metals (the nickel-copper system): Japanese Journal of Physics, v. 8, p. 109-113.

Mehrer, H., 2007, Diffusion in Solids-Fundamentals, Methods, Materials, Diffusion-Controlled Processes: Berlin, Germany, Springer Series in Solid State Science, v. 155, 672 p.

Neusser, G., Abart, R., Fischer, F. D., Harlov, D., and Norberg, N., 2012, Experimental Na/K exchange between alkali feldspar and an $\mathrm{NaCl}-\mathrm{KCl}$ salt melt: chemically induced fracturing and element partitioning: Contributions to Mineralogy and Petrology, v. 164, n. 2, p. 341-358, http://dx.doi.org/ $10.1007 / \mathrm{s} 00410-012-0741-9$

Norberg, N., Neusser, G., Wirth, R., and Harlov, D., 2012, Microstructural evolution during experimental albitization of K-rich alkali feldspar: Contributions to Mineralogy and Petrology, v. 162, n. 3, p. 531-546, http://dx.doi.org/10.1007/s00410-011-0610-y

Nye, J., 1957, Physical Properties of Crystals: Oxford, Clarendon Press, 322 p.

Petrishcheva, E., and Abart, R., 2009, Exsolution by spinodal decomposition I: Evolution equation for binary mineral solutions with anisotropic interfacial energy: American Journal of Science, v. 309, n. 6, p. 431-449, http://dx.doi.org/10.2475/06.2009.01

2012, Exsolution by spinodal decomposition in multicomponent mineral solutions: Acta Materialia, v. 60 , n. 15 , p. 5481-5493, http://dx.doi.org/10.1016/j.actamat.2012.07.006

Petrović, R., ms, 1972, Alkali ion diffusion in alkali feldspars: New Haven, Connecticut, Yale University, Ph. D. Dissertation, $262 \mathrm{p}$.

Riley, T., and Bailey, D., 2003, Barium-rich sanidine megacrysts from the West Eifel (Germany): Neues Jahrbuch für Mineralogie-Monatshefte, v. 41, p. 18-30, http://dx.doi.org/10.1127/0028-3649/2003/ 2003-0018

Schäffer, A. K., Jaepel, T., Zaefferer, S., Abart, R., and Rhede, D., 2014a, Lattice strain across Na-K interdiffusion fronts in alkali feldspar: an electron back-scatter diffraction study: Physics and Chemistry of Minerals, http://dx.doi.org/10.1007/s00269-014-0692-y

Schäffer, A., Petrishcheva, E., Halber, G., Abart, R., Rhede, D., and Giester, G., 2014b, Sodium-potassium interdiffusion in potassium-rich alkali feldspar II: Composition- and temperature-dependence obtained from cation exchange experiments: American Journal of Science, v. 314, n. 9, p. 1303-1318, http:// dx.doi.org/10.2475/09.2014.03

Scheidl, K., Schäffer, A., Petrishcheva, E., Habler, G., Fischer, F., Schreuer, J., and Abart, R., 2014, Chemically induced fracturing in alkali feldspar: Physics and Chemistry of Minerals, v. 41, n. 1, p. 1-16, http://dx.doi.org/10.1007/s00269-013-0617-1

Svoboda, J., Fischer, F., and Fratzl, P., 2002, Diffusion in multi-component systems with no or dense sources and sinks for vacancies: Acta Materialia, v. 50, n. 6, p. 1369-1381, http://dx.doi.org/10.1016/S13596454(01)00443-8

Voll, G., Evangelakakis, C., and Kroll, H., 1994, Revised two-feldspar geothermometry applied to Sri Lankan feldspars: Precambrian Research, v. 66, n. 1-4, p. 351-377, http://dx.doi.org/10.1016/0301-9268(94) 90058-2

Weitz, G., 1972, Die Struktur des Sanidins bei verschiedenen Ordnungsgraden: Zeitschrift für Kristallographie, v. 136, n. 5-6, p. 418-426, http://dx.doi.org/10.1524/zkri.1972.136.5-6.418

Yund, R., 1984, Alkali Feldspar exsolution: Kinetics and dependence on alkali interdiffusion, in Brown, W. L, editor, Feldspars and Feldspathoids-Structures, Properties and Occurrences: Dordrecht, Holland, D. Reidel Publishing Company, p. 281-316. 